\title{
Assessment of dietary habits and nutritional status of depressive patients, depending on place of residence
}

\author{
Ewa Stefanska', Agnieszka Wendołowicz', Urszula Cwalina², Urszula Kowzan³, \\ Beata Konarzewska ${ }^{3}$, Agata Szulc ${ }^{3}$, Lucyna Ostrowska' \\ ${ }^{1}$ Department of Dietetics and Clinical Nutrition, Medical University of Bialystok, Poland \\ ${ }^{2}$ Department of Medical Statistics and Informatics, Medical University of Bialystok, Poland \\ ${ }^{3}$ Department of Psychiatry, Medical University of Bialystok, Poland
}

Stefanska E, Wendołowicz A, Cwalina U, Kowzan U, Konarzewska B, Szulc A, Ostrowska L. Assessment of dietary habits and nutritional status of depressive patients,depending on place of residence. Ann Agric Environ Med. 2017; 24(4): 581-586. doi: 10.5604/12321966.1233554

\begin{abstract}
Introduction and objectives. An increased incidence of depressive disorders observed in recent years in the Polish and world population is a serious health problem. The aim of the study was to compare dietary habits and nutritional status of patients with recurrent depressive disorders, depending on their place of residence. Their impact on selected metabolic parameters was also considered.

Materials and method. The study group comprised 96 women and 84 men reporting to the Outpatient Mental Health Clinic at the Department of Psychiatry, Medical University of Bialystok in north-eastern Poland. The average age of the women was $46.7 \pm 11.7$ years, and of men $47.0 \pm 11.3$ years. In the quantitative assessmentof diets, 24 -hour food recall interviews were conducted. Assessment of the nutritional status of the respondents consisted of anthropometric measurements, body composition analysis and biochemical parameters.

Results. It was show that the diets of female urban inhabitants were characterized by a significantly lower energy value and total fat content, compared to their rural counterparts. The food rations of men living in the city had a significantly higher energy value, protein content and total FAT, compared to rural residents. It was also noted that urban residents of both genders were characterized by a lower percentage of body fat, both visceral and subcutaneous (women), and a higher water content than rural residents.

Conclusions. The study showed dietary errors in all compared groups, regardless of place of residence, which was reflected in the nutritional status of the respondents. The results also indicated that during the declared change in dietary habits, the treatment of depressive patients should include dietary instructions in order to ensure an optimum supply of nutrients.
\end{abstract}

\section{Key words}

dietary habits, depression, urban area, rural area

\section{INTRODUCTION}

Depression is a psychosomatic disorder, affecting more than 121 million people worldwide [1]. The estimated number of adults suffering from depressive disorders in Poland amounts to $1.2-1.5$ million people. The disorder is more common in women than in men [2]. In the USA, depressive disorders affect $21.3 \%$ of women and $12.7 \%$ of men [1]. According to American research, about $20-30 \%$ of patients aged $18-54$ years admitted by GPs display various depressive symptoms which increase significantly for those over 55 years of age[3].

The growing incidence of depressive disorders in the Polish and world population is a serious problem, both for adults and young people, and is one of the causes of early disability [2]. It has been estimated that by 2020 , depression will be among the three main health problems worldwide [4]. In many studies, depression has appeared to be an independent predictor of cardiovascular diseases, including coronary heart disease, to facilitate body weight gain and increase the percentage of patients with overweight or obesity, especially

Addres for correspondence: Ewa Stefanska, Department of Dietetics and Clinical Nutrition, Medical University of Bialystok, Poland

E-mail:estef@umb.edu.pl

Received: 29 April 2014; accepted: 17 November 2014; first published on February 017 abdominal obesity and accumulation of metabolically active visceral adipose tissue (VAT) $[2,5,6,7]$. Depression has also been shown to occur in approximately $30 \%$ of patients with type 1 and type 2 diabetes [8].

As research shows, some socio-demographic factors (including age, education, marital status or place of residence) may differentiate the occurrence of depressive disorders $[1,4,9,10,11]$. However, the few studies that analyzed the incidence of depression depending on the place of residence have demonstrated ambiguous results. Peen et al. found that psychosomatic disorders, including depression, occurred more frequently in city patients; other researchers found a higher incidence of depression among residents of rural areas than the urban areas $[4,10]$.

\section{OBJECTIVES}

The aim of the study is to compare dietary habits and the nutritional status (as well as their impact on selected metabolic parameters) of patients with recurrent depressive disorder, depending on their place of residence. 


\section{MATERIALS AND METHOD}

The study group comprised 96 women and 84 men (48 female and 44 male residents of Bialystok in north-eastern Poland, and 48 female and 40 male rural residents) reporting to the Outpatient Mental Health Clinic at the Department of Psychiatry, Medical University of Bialystok in 2012-2013. The average age of female city inhabitants was $46.3 \pm 14.1$ years, and of village residents $49.2 \pm 8.3$ years. The average age of men living in the city was $45.4 \pm 10.9$ and in the village $47.3 \pm 12.1$ years. The study group included patients with the diagnosis of recurrent depressive disorders (ICD-10), lasting up to five years, with a current episode of depression not lasting longer than a month [12].

Furthermore, the current antidepressant treatment lasted no longer than one month, and consisted of one of the following drugs: paroxetine, sertraline, venlafaxine, citalopram, mirtazapine, escitalopram, and one sedative drug used in emergency. The disease course was assessed on the basis of medical history and available documentation. The Hamilton Depression Rating Scale (17-point version) and Beck Self-Esteem Scale [13, 14] were used to rate the severity of depression.

Patients who did not have any chronic metabolic diseases were enrolled to the study and informel about the purpose and methodology. Each patient gave written consent to participate. The studywas approved by the local Bioethics Committee (No. RI-002/325/2011).

Nutritional status. Assessment of the nutritional status of the respondents consisted of body weight measurement, height measurement, and Body Mass Index (BMI). Body composition analysis was performed by bioelectrical impedancje using a Maltron 920-2BioScan analyzer (MaltronInternational Ltd.). Measurements of body composition were carried out in the morning hours on an empty stomach, as recommended. Patients declared no consumption of coffee or Rother caffeinated products, strong tea, and alkohol for at least 24 hours prior to testing.

The percentage content of fat in the body (FM, fat mass), fat mass in kilograms, depth of subcutaneous adipose tissue area (SAT, Subcutaneous Fat), depth of fat in the visceral part (VAT, Visceral Fat), percentage of fat free body mass (FFM, fat free mass), lean tissue content expressed in kilograms, the percentage content of total body water (TBW), extracellularwater (ECW) and intracellular water (ICW) were assessed in the hospital laboratory. In terms of biochemical parameters of the nutritional status, the following were assessed: blood lipid profile (total cholesterol, LDL-cholesterol, HDL-cholesterol and triglycerides), blood hemoglobin and glucose levels. The values obtained were referred to the currently applicable standards $[15,16]$.

Dietary intake assessment. Dietary data were collected using a questionnaire designer in the Department of Dietetics and Clinical Nutrition at the Medical University of Białystok. In the quantitative assessment of diets, 24-hour food recall interviews were conducted, including 3 weekdays and 1 weekend day, and the results were averaged. The assessed patients did not use any additional vitamin-mineral supplementation. Portion sizes of food products were estimated using the 'Photo Album of Food Products' elaborated in the Food and Nutrition Institute (IZZ) in Warsaw [17].
The nutritional value of daily food rations was analyzed using computer programme Diet5.0 (IZZ, Warsaw), taking into account the loss of nutrients during food processing The nutrition standards for the Polish population [18] were used to evaluate the compatibility of the selected nutrients with recommendations.

Statistical analysis. Performed Rusing Statistica10.0, StatSoftInc. Normal distribution of numerical variables was determined using the Shapiro-Wilk test. Student's t-test was used to compare variables in normal distribution; the nonparametric Mann-Whitney U test was applied for abnormal distribution. To assess the relationship between the nominal characteristics, the $\chi^{2}$ test was used. Results were considered significant at $\mathrm{p}<0.05$.

\section{RESULTS}

Among the female respondents from urban areas, 45.8\% had a single episode of depression, $16.7 \%$ of women reported $2-4$ episodes and $37.5 \%$ more than 4 episodes. In the group of female village residents, $31.2 \%$ had a single episode of depression, $20.8 \% 2-4$ episodes and $48 \%$ more than 4 episodes. In the group of male urban residents, a single episode of depression was reported by $59 \%, 2-4$ episodes by $13.7 \%$, and more than 4 episodes by $27.3 \%$ of the patients. Among the male rural residents, $32.5 \%$ had a single episode of depression, $30.0 \%$ reported $2-4$ episodes and $37.5 \%$ more than 4 episodes.

Table 1 shows the general characteristics of study patients. Female Urban residents had a significantly lower mean value of body weight $(p=0,0047)$, waist circumference $(p=0,0014)$ and $\operatorname{BMI}(\mathrm{p}=0,0027)$, compared to their rural counterparts. No such differences were found among the men. The marital status turned out to be a significantly differentiating factor only in women (significantly more female urban residents with depression were unmarried or divorced, $\mathrm{p}=0,0128$ ). According to the level of education, there were more respondents with primary education, both among men $(\mathrm{p}=0,0001)$ and women( $\mathrm{p}=0,0060)$, with depression living in villages, compared to those living inthe city.

Table 2 shows the selected parameters of the nutritional status of patients according to gender and place of residence. In the compared groups of women, fat content was significantly higher for women from rural areas than from city $(p=0,0013)$. On the other hand, urban female residents had statistically higher fat free mass $(\%)(\mathrm{p}=0,0023)$, total body water $(\%)(\mathrm{p}=0,0031)$ and extracellular water $(\%)$ $(\mathrm{p}=0,0228)$ and ECW/ICW ratio $(\mathrm{p}=0,0386)$. Urban female residents were characterized by lower average visceral and subcutaneous adipose tissue content compared to their urban counterparts, although the differences were not statistically significant.

Male residents of the city were characterized by lower total body fat, visceral fat, intracellular water and higher content of fat free mass, subcutaneous fat, total body water and extracellular water content in comparison with village inhabitants (differences not statistically significant). In the two groups of women, despite lack of statistically significant differences, those from the city (with the exception of the average total cholesterol levels) were characterized by lipid parameters closest to current standards. 
Table 1. General characteristics of respondents

\begin{tabular}{|c|c|c|c|c|c|}
\hline \multirow[b]{2}{*}{ Variables } & \multirow{2}{*}{$\begin{array}{l}\text { Param- } \\
\text { eter }\end{array}$} & \multicolumn{2}{|c|}{ Women $(n=96)$} & \multicolumn{2}{|c|}{ Men $(n=84)$} \\
\hline & & $\begin{array}{l}\text { Urban } \\
(n=48)\end{array}$ & $\begin{array}{l}\text { Rural } \\
(n=48)\end{array}$ & $\begin{array}{l}\text { Urban } \\
(n=44)\end{array}$ & $\begin{array}{l}\text { Rural } \\
(\mathrm{n}=40)\end{array}$ \\
\hline \multirow{2}{*}{ Body height $(\mathrm{cm})$} & Mean \pm SD & $163.4 \pm 6.1$ & $162.9 \pm 5.5$ & $177.3 \pm 7.6$ & $174.2 \pm 4.2$ \\
\hline & Median & 163.3 & 164.0 & 179.0 & 173.0 \\
\hline \multirow{2}{*}{ Body weight (kg) } & Mean \pm SD & $65.9 \pm 11.6^{* *}$ & $74.9 \pm 15.2^{* *}$ & $89.8 \pm 13.5$ & $86.3 \pm 13.6$ \\
\hline & Median & 65.5 & 76.0 & 87.5 & 90.0 \\
\hline \multirow{2}{*}{$\begin{array}{l}\text { Waist } \\
\text { circumference }(\mathrm{cm})\end{array}$} & Mean \pm SD & $89.1 \pm 12.1^{* *}$ & $99.0 \pm 14.3^{* *}$ & $102.2 \pm 8.9$ & $102.8 \pm 9.7$ \\
\hline & Median & 91.0 & 100.5 & 102.0 & 107.0 \\
\hline \multirow{2}{*}{$\begin{array}{l}\text { Body mass index } \\
\left(\mathrm{kg} / \mathrm{m}^{2}\right)\end{array}$} & Mean \pm SD & $24.7 \pm 4.5^{* *}$ & $28.2 \pm 5.2^{* *}$ & $28.5 \pm 3.2$ & $28.4 \pm 4.1$ \\
\hline & Median & 24.9 & 28.4 & 28.1 & 29.5 \\
\hline \multicolumn{6}{|l|}{ Body mass index } \\
\hline Normal weight & $(\%)$ & $50.0^{* *}$ & $31.6^{* *}$ & 4.6 & 17.5 \\
\hline Overweight & (\%) & 39.5 & 23.7 & 63.6 & 32.5 \\
\hline Obese & (\%) & 10.5 & 44.7 & 31.8 & 50.0 \\
\hline \multicolumn{6}{|l|}{ Marital status } \\
\hline Single & (\%) & $21.1^{*}$ & $13.2^{*}$ & 22.7 & 17.5 \\
\hline Married & (\%) & 52.6 & 71.0 & 72.7 & 65.0 \\
\hline Divorced & (\%) & 23.7 & 2.6 & 4.6 & 17.5 \\
\hline Widow & (\%) & 2.6 & 13.2 & 0.0 & 0.0 \\
\hline \multicolumn{6}{|l|}{ Education } \\
\hline Primary & $(\%)$ & $18.4^{* *}$ & $52.6^{* *}$ & $36.4^{* * *}$ & $82.5^{* * *}$ \\
\hline Secondary & (\%) & 60.5 & 39.5 & 36.4 & 17.5 \\
\hline University & (\%) & 21.1 & 7.9 & 27.2 & 0.0 \\
\hline \multirow{2}{*}{ HAM-D score } & Mean $\pm S D$ & $13.6 \pm 6.9$ & $13.5 \pm 6.1$ & $12.1 \pm 6.7$ & $10.7 \pm 5.8$ \\
\hline & Median & 14.5 & 12.5 & 12.0 & 11.0 \\
\hline \multirow{2}{*}{ Beck score } & Mean \pm SD & $23.9 \pm 12.1$ & $24.9 \pm 12.5$ & $18.2 \pm 4.8$ & $13.6 \pm 4.6$ \\
\hline & Median & 25.5 & 28.0 & 18.0 & 14.0 \\
\hline
\end{tabular}

statistically significant differences within gender groups compared: * $-<0.05 ; * * 0<0.01$ $* * *-p<0.001$

However, higher levels of total cholesterol $(\geq 190 \mathrm{mg} / \mathrm{dl})$ were reported in $29.2 \%$ of female cityresidentsand $42 \%$ of female villageinhabitants. Decreased levels ofHDLcholesterol $(\leq 50 \mathrm{mg} / \mathrm{dL})$ appeared in $39.6 \%$ of city women and $60.4 \%$ of those from countryside.Higher levels ofLDLcholesterol $(>115 \mathrm{mg} / \mathrm{dl})$ were noted in $58.3 \%$ of urban women and $69 \%$ of village women.Among the female residents, assessment of glycaemia on an empty stomach showed higherglucose levels ( $\geq 100 \mathrm{mg} / \mathrm{dl})$ in $35 \%$ of women from the city and in $47.9 \%$ of those from countryside.It was also shownthatreduced levels of blood hemoglobin $(<12 \mathrm{~g} / \mathrm{dl})$ occurred in $16.7 \%$ of women from the city and $8.3 \%$ of women from countryside.Significantlyhigher levels oftriglyceridesin blood serum $(p=0,0084)$ were demonstrated by city male inhabitantsas comparedto male villagers(elevated levels oftriglycerideswere found in $63.6 \%$ of the urban and in $40 \%$ of the rural population).

At the same time, elevated levels oftotal cholesterol were reported innearly $70 \%$ of men in both study groups. Decreased levels ofHDL-cholesterol $(\leq 40 \mathrm{mg} / \mathrm{dl})$ were noted in $36.4 \%$ of urban and in $40 \%$ of rural residents. Elevated levels ofLDL-cholesterol were present inapproximately $60 \%$ of patients in both male groups.In addition,elevatedglucose valueswere recordedin $47.7 \%$ of men from the city, and $62.5 \%$ of men from the village. It was also shownthatreduced levels ofhemoglobin in the blood $(<14 \mathrm{~g} / \mathrm{dl})$ occurred in $13.6 \%$ of men fromthe city, and $10 \%$ of men from the village.

Table 3 shows mean energy value in daily food rations and average intake of main nutrients by patients. In the study groups of women, statistically significant differences
Table 2. Chosen parameters of nutritional status of patients according to gender and place of residence

\begin{tabular}{|c|c|c|c|c|c|}
\hline \multirow[b]{2}{*}{ Variables } & \multirow{2}{*}{$\begin{array}{l}\text { Param- } \\
\text { eter }\end{array}$} & \multicolumn{2}{|c|}{ Women $(n=96)$} & \multicolumn{2}{|c|}{ Men $(n=84)$} \\
\hline & & $\begin{array}{l}\text { Urban } \\
(\mathrm{n}=48)\end{array}$ & $\begin{array}{c}\text { Rural } \\
(n=48)\end{array}$ & $\begin{array}{l}\text { Urban } \\
(\mathrm{n}=44)\end{array}$ & $\begin{array}{c}\text { Rural } \\
(n=40)\end{array}$ \\
\hline \multirow{2}{*}{$\begin{array}{l}\text { Fat mass } \\
\text { (FM) (\%) }\end{array}$} & Mean $\pm S D$ & $30.3 \pm 9.3$ & $37.2 \pm 8.1$ & $29.0 \pm 5.0$ & $31.2 \pm 8.2$ \\
\hline & Median & $31.9 * *$ & $39.3^{* *}$ & 28.7 & 30.7 \\
\hline \multirow{2}{*}{$\begin{array}{l}\text { Fat mass } \\
(\mathrm{kg})\end{array}$} & Mean \pm SD & $21.1 \pm 9.3$ & $28.9 \pm 10.7$ & $25.9 \pm 6.6$ & $27.7 \pm 9.7$ \\
\hline & Median & $21.9^{* *}$ & $30.5^{* *}$ & 24.8 & 27.8 \\
\hline \multirow{2}{*}{$\begin{array}{l}\text { Fat free } \\
\text { mass (FFM) } \\
(\%)\end{array}$} & Mean \pm SD & $69.5 \pm 9.4$ & $62.8 \pm 8.1$ & $70.9 \pm 5.0$ & $68.7 \pm 8.2$ \\
\hline & Median & $67.1 * *$ & $60.7^{* *}$ & 70.3 & 69.2 \\
\hline \multirow{2}{*}{$\begin{array}{l}\text { Fat free } \\
\text { mass }(\mathrm{kg})\end{array}$} & Mean \pm SD & $45.1 \pm 4.1$ & $46.4 \pm 5.7$ & $62.5 \pm 11.1$ & $58.4 \pm 5.7$ \\
\hline & Median & 45.5 & 46.2 & 63.9 & 59.3 \\
\hline \multirow{2}{*}{$\begin{array}{l}\text { Visceral fat } \\
\left(\text { VAT) }\left(\mathrm{cm}^{2}\right)\right.\end{array}$} & Mean \pm SD & $244.5 \pm 206.3$ & $275.7 \pm 219.2$ & $394.3 \pm 328.4$ & $435.5 \pm 417.3$ \\
\hline & Median & 174.0 & 208.0 & 260.5 & 195.0 \\
\hline \multirow{2}{*}{$\begin{array}{l}\text { Subcuta- } \\
\text { neous fat } \\
(\mathrm{SAT})\left(\mathrm{cm}^{2}\right)\end{array}$} & Mean $\pm S D$ & $105.3 \pm 33.8$ & $124.2 \pm 44.5$ & $127.3 \pm 56.5$ & $124.4 \pm 36.7$ \\
\hline & Median & 89.0 & 118.5 & 112.0 & 104.5 \\
\hline \multirow{2}{*}{ VAT/SAT } & Mean \pm SD & $2.3 \pm 1.7$ & $2.1 \pm 1.3$ & $3.2 \pm 2.673$ & $3.4 \pm 3.8$ \\
\hline & Median & 1.7 & 1.7 & 2.210 & 1.950 \\
\hline \multirow{2}{*}{$\begin{array}{l}\text { Total body } \\
\text { water } \\
(\text { TBW)(\%) }\end{array}$} & Mean \pm SD & $52.0 \pm 5.2$ & $48.7 \pm 5.5$ & $53.1 \pm 3.2$ & $51.5 \pm 4.3$ \\
\hline & Median & $51.0^{* *}$ & $47.6^{* *}$ & 53.2 & 51.8 \\
\hline \multirow{2}{*}{$\begin{array}{l}\text { Extra- } \\
\text { cellular } \\
\text { water } \\
(\text { ECW) (\%) }\end{array}$} & Mean \pm SD & $48.3 \pm 3.8$ & $45.7 \pm 5.7$ & $43.1 \pm 2.1$ & $42.5 \pm 1.4$ \\
\hline & Median & $47.8^{*}$ & $46.7^{*}$ & 42.8 & 43.3 \\
\hline \multirow{2}{*}{$\begin{array}{l}\text { Intra- } \\
\text { cellular } \\
\text { water (ICW) } \\
(\%)\end{array}$} & Mean $\pm S D$ & $51.7 \pm 3.9$ & $53.3 \pm 2.3$ & $56.8 \pm 2.1$ & $57.4 \pm 1.4$ \\
\hline & Median & 52.2 & 53.1 & 57.1 & 56.7 \\
\hline \multirow{2}{*}{$\mathrm{ECW} / \mathrm{ICW}$} & Mean \pm SD & $0.94 \pm 0.2$ & $0.87 \pm 0.07$ & $0.76 \pm 0.06$ & $0.74 \pm 0.04$ \\
\hline & Median & $0.91 *$ & $0.88^{*}$ & 0.750 & 0.763 \\
\hline \multirow{2}{*}{$\begin{array}{l}\text { Total } \\
\text { cholesterol } \\
(\mathrm{mg} / \mathrm{dl})\end{array}$} & Mean \pm SD & $216.1 \pm 50.5$ & $207.4 \pm 34.9$ & $220.6 \pm 34.6$ & $213.0 \pm 47.0$ \\
\hline & Median & 197.5 & 204 & 212.0 & 226.0 \\
\hline \multirow{2}{*}{$\begin{array}{l}\mathrm{HDL}- \\
\text { cholesterol } \\
(\mathrm{mg} / \mathrm{dl})\end{array}$} & Mean \pm SD & $55.8 \pm 11.9$ & $52.6 \pm 13.2$ & $46.7 \pm 10.8$ & $39.8 \pm 4.4$ \\
\hline & Median & 57.0 & 52.0 & 42.0 & 38.0 \\
\hline \multirow{2}{*}{$\begin{array}{l}\text { LDL- } \\
\text { cholesterol } \\
(\mathrm{mg} / \mathrm{dl})\end{array}$} & Mean \pm SD & $124.8 \pm 41.1$ & $127.7 \pm 30.6$ & $134.4 \pm 38.3$ & $147.2 \pm 47.4$ \\
\hline & Median & 121.0 & 127.0 & 135.5 & 157.0 \\
\hline \multirow{2}{*}{$\begin{array}{l}\text { Tri- } \\
\text { glycerides } \\
(\mathrm{mg} / \mathrm{dl})\end{array}$} & Mean $\pm S D$ & $131.2 \pm 100.6$ & $135.1 \pm 84.6$ & $197.1 \pm 103.1$ & $132.5 \pm 17.5$ \\
\hline & Median & 104.5 & 103.0 & $180.0^{* *}$ & $138.0^{* *}$ \\
\hline \multirow{2}{*}{$\begin{array}{l}\text { Glucose } \\
\text { (mg/dl) }\end{array}$} & Mean \pm SD & $103.6 \pm 13.8$ & $106.5 \pm 11.9$ & $102.1 \pm 14.1$ & $106.0 \pm 15.3$ \\
\hline & Median & 101.0 & 107.5 & 100.0 & 105.0 \\
\hline \multirow{2}{*}{$\begin{array}{l}\text { Haemo- } \\
\text { globin (g/ } \\
\text { dl) }\end{array}$} & Mean \pm SD & $13.2 \pm 1.48$ & $13.2 \pm 1.1$ & $14.6 \pm 0.8$ & $14.4 \pm 1.4$ \\
\hline & Median & 13.7 & 13.4 & 14.6 & 14.9 \\
\hline
\end{tabular}

statistically significant differences within gender groups compared: ${ }^{*}-<0.05$; ${ }^{*}-p<0.01$; $* * *-p<0.001$

were demonstrated in supply of energy $(\mathrm{p}=0,0271)$, total fat $(\mathrm{p}=0,0294)$ and saturated fatty acids $(\mathrm{p}=0,0413)$, where the intake was significantly lower in city residents as compared to rural inhabitants.

Men demonstrated statistically significant differences in the supply of energy $(\mathrm{p}=0,0212)$, total protein $(\mathrm{p}=0.0019)$ and total fat $(\mathrm{p}=0,0180)$, whose intake was significantly higher in the urban population compared to the rural one. 
Table 3. Average energy value and consumption of selected nutrients in the groups of patients compared by gender and place of residence

\begin{tabular}{|c|c|c|c|c|c|}
\hline \multirow[b]{2}{*}{ Variables } & \multirow{2}{*}{$\begin{array}{l}\text { Param- } \\
\text { eter }\end{array}$} & \multicolumn{2}{|c|}{ Women $(n=96)$} & \multicolumn{2}{|c|}{ Men $(n=84)$} \\
\hline & & $\begin{array}{l}\text { Urban } \\
(n=48)\end{array}$ & $\begin{array}{l}\text { Rural } \\
(n=48)\end{array}$ & $\begin{array}{l}\text { Urban } \\
(n=44)\end{array}$ & $\begin{array}{l}\text { Rural } \\
(n=40)\end{array}$ \\
\hline \multirow{2}{*}{$\begin{array}{l}\text { Energy } \\
\text { (kcal/day) } \\
{[\% \text { norm] }}\end{array}$} & Mean $\pm S D$ & $\begin{array}{c}1497.2 \pm \\
646.4 \\
{[80.9]}\end{array}$ & $\begin{array}{c}1718.0 \pm \\
497.4 \\
{[92.8]}\end{array}$ & $\begin{array}{c}2069.4 \pm \\
676.6 \\
{[84.5]}\end{array}$ & $\begin{array}{c}1691.3 \pm \\
511.7 \\
{[69]}\end{array}$ \\
\hline & Median & $\begin{array}{c}1308.2^{*} \\
{[70.7]}\end{array}$ & $\begin{array}{c}1690.4^{*} \\
{[91.3]}\end{array}$ & $\begin{array}{c}1925.0^{*} \\
{[78.6]}\end{array}$ & $\begin{array}{c}1629.2^{*} \\
{[66.4]}\end{array}$ \\
\hline \multirow{2}{*}{$\begin{array}{l}\text { Total } \\
\text { protein (g/ } \\
\text { day) } \\
\text { [\% norm] }\end{array}$} & Mean \pm SD & $\begin{array}{c}59.8 \pm 26.6 \\
{[130]}\end{array}$ & $\begin{array}{c}64.9 \pm 20.4 \\
{[141]}\end{array}$ & $\begin{array}{c}74.5 \pm 24.7 \\
{[122]}\end{array}$ & $\begin{array}{c}59.3 \pm 11.9 \\
{[97.2]}\end{array}$ \\
\hline & Median & $\begin{array}{c}53.0 \\
{[115]}\end{array}$ & $\begin{array}{c}62.1 \\
{[135]}\end{array}$ & $\begin{array}{c}70.9 * * \\
{[116]}\end{array}$ & $\begin{array}{l}54.8^{* *} \\
{[89.8]}\end{array}$ \\
\hline \multirow{2}{*}{$\begin{array}{l}\text { Total fat (g/ } \\
\text { day) } \\
{[\% \text { norm }]}\end{array}$} & $\begin{array}{l}\text { Mean } \pm S D \\
{[\% \text { norm] }}\end{array}$ & $\begin{array}{c}51.5 \pm 28.0 \\
{[83]}\end{array}$ & $\begin{array}{c}63.1 \pm 25.5 \\
{[101.8]}\end{array}$ & $\begin{array}{c}84.6 \pm 36.8 \\
{[103.7]}\end{array}$ & $\begin{array}{c}66.7 \pm 29.5 \\
{[81.7]} \\
\end{array}$ \\
\hline & Median & $\begin{array}{c}44.1^{*} \\
{[71]}\end{array}$ & $\begin{array}{l}60.2^{*} \\
{[97.1]}\end{array}$ & $\begin{array}{l}76.2^{*} \\
{[93.4]}\end{array}$ & $\begin{array}{l}56.9^{*} \\
{[69.7]}\end{array}$ \\
\hline \multirow{2}{*}{$\begin{array}{l}\text { Saturated } \\
\text { fatty acids } \\
\text { (SFA) (g/ } \\
\text { day) } \\
\text { [\% norm] }\end{array}$} & Mean $\pm S D$ & $\begin{array}{c}21.5 \pm 11.4 \\
{[131]}\end{array}$ & $\begin{array}{c}27.2 \pm 13.6 \\
{[165.8]}\end{array}$ & $\begin{array}{c}38.1 \pm 17.7 \\
{[174.8]}\end{array}$ & $\begin{array}{c}29.1 \pm 13.5 \\
{[133.5]}\end{array}$ \\
\hline & Median & $\begin{array}{l}19.5^{*} \\
{[119]}\end{array}$ & $\begin{array}{c}26.0^{*} \\
{[158.5]}\end{array}$ & $\begin{array}{c}34.2 \\
{[156.9]}\end{array}$ & $\begin{array}{c}25.8 \\
{[118.3]}\end{array}$ \\
\hline \multirow{2}{*}{$\begin{array}{l}\text { Monoun- } \\
\text { saturated } \\
\text { fatty acids } \\
\text { (MUFA) (g/ } \\
\text { day) } \\
\text { [\% norm] }\end{array}$} & Mean $\pm S D$ & $\begin{array}{c}20.1 \pm 12.0 \\
{[65.2]}\end{array}$ & $\begin{array}{c}24.4 \pm 10.5 \\
{[79.2]}\end{array}$ & $\begin{array}{c}32.5 \pm 14.4 \\
{[79.6]}\end{array}$ & $\begin{array}{c}26.6 \pm 12.1 \\
{[65.2]}\end{array}$ \\
\hline & Median & $\begin{array}{c}17.0 \\
{[55.2]}\end{array}$ & $\begin{array}{c}22.7 \\
{[73.7]}\end{array}$ & $\begin{array}{l}29.3 \\
{[72]}\end{array}$ & $\begin{array}{l}23.5 \\
{[57.6]}\end{array}$ \\
\hline \multirow{2}{*}{$\begin{array}{l}\text { Polyun- } \\
\text { saturated } \\
\text { fatty acids } \\
\text { (PUFA) (g/ } \\
\text { day) } \\
\text { [\% norm] } \\
\end{array}$} & Mean $\pm S D$ & $\begin{array}{c}6.2 \pm 5.1 \\
{[43]}\end{array}$ & $\begin{array}{c}5.9 \pm 2.9 \\
{[50]}\end{array}$ & $\begin{array}{c}7.4 \pm 4.0 \\
{[38.9]}\end{array}$ & $\begin{array}{c}5.6 \pm 2.3 \\
{[29.5]}\end{array}$ \\
\hline & Median & $\begin{array}{c}4.4 \\
{[30.5]}\end{array}$ & $\begin{array}{c}5.4 \\
{[37.5]}\end{array}$ & $\begin{array}{c}6.4 \\
{[33.7]}\end{array}$ & $\begin{array}{c}4.9 \\
{[25.8]}\end{array}$ \\
\hline \multirow{2}{*}{$\begin{array}{l}\text { Cholesterol } \\
\text { (mg/day) } \\
{[\% \text { norm }]}\end{array}$} & Mean $\pm S D$ & $\begin{array}{c}207.2 \pm 68.3 \\
{[69]}\end{array}$ & $\begin{array}{c}263.8 \pm 186.5 \\
{[88]}\end{array}$ & $\begin{array}{c}323.8 \pm 189.7 \\
{[108]}\end{array}$ & $\begin{array}{c}201.7 \pm 77.3 \\
{[67]}\end{array}$ \\
\hline & Median & $\begin{array}{l}154.7 \\
{[51.5]}\end{array}$ & $\begin{array}{l}192.7 \\
{[65.7]}\end{array}$ & $\begin{array}{l}265.6 \\
{[88.5]}\end{array}$ & $\begin{array}{l}190.1 \\
{[63.4]}\end{array}$ \\
\hline \multirow{2}{*}{$\begin{array}{l}\text { Carbo- } \\
\text { hydrates } \\
\text { (g/day) } \\
{[\% \text { norm] }}\end{array}$} & Mean \pm SD & $\begin{array}{c}212.6 \pm 88,2 \\
{[76.6]}\end{array}$ & $\begin{array}{c}239.6 \pm 73.7 \\
{[86.3]}\end{array}$ & $\begin{array}{c}269.3 \pm 87.2 \\
{[73.3]} \\
\end{array}$ & $\begin{array}{c}210.5 \pm 71.4 \\
{[57.3]}\end{array}$ \\
\hline & Median & $\begin{array}{l}193.6 \\
{[69.8]}\end{array}$ & $\begin{array}{l}227.3 \\
{[81.9]}\end{array}$ & $\begin{array}{l}255.0 \\
{[69.4]}\end{array}$ & $\begin{array}{l}189.2 \\
{[51.5]}\end{array}$ \\
\hline \multirow{2}{*}{$\begin{array}{l}\text { Fiber } \\
\text { g/day) } \\
{[\% \text { norm] }}\end{array}$} & Mean \pm SD & $\begin{array}{c}16.0 \pm 5.3 \\
{[53.3]}\end{array}$ & $\begin{array}{c}18.1 \pm 8.3 \\
{[60.3]}\end{array}$ & $\begin{array}{c}18.8 \pm 6.3 \\
{[62.6]}\end{array}$ & $\begin{array}{c}13.5 \pm 6.2 \\
{[45]}\end{array}$ \\
\hline & Median & $\begin{array}{c}15.1 \\
{[50.3]}\end{array}$ & $\begin{array}{l}16.8 \\
{[56]}\end{array}$ & $\begin{array}{l}18.9 \\
{[63]}\end{array}$ & $\begin{array}{c}11.3 \\
{[37.7]}\end{array}$ \\
\hline \multirow{2}{*}{$\begin{array}{l}\text { Protein } \\
(\% \mathrm{E})\end{array}$} & Mean $\pm S D$ & $16.5 \pm 3.3$ & $15.1 \pm 2.6$ & $15.1 \pm 3.9$ & $15.1 \pm 3.7$ \\
\hline & Median & $16.0^{*}$ & $14.5^{*}$ & 14.3 & 14.9 \\
\hline \multirow{2}{*}{$\begin{array}{l}\text { Total Fat } \\
(\% \mathrm{E})\end{array}$} & Mean $\pm S D$ & $29.4 \pm 7.0$ & $32.1 \pm 8.4$ & $35.1 \pm 7.0$ & $34.8 \pm 7.3$ \\
\hline & Median & 28.6 & 32.4 & 37.0 & 36.9 \\
\hline \multirow{2}{*}{$\begin{array}{l}\text { Carbo- } \\
\text { hydrates } \\
(\% \mathrm{E})\end{array}$} & Mean $\pm S D$ & $54.0 \pm 7.1$ & $52.7 \pm 7.4$ & $49.7 \pm 7.0$ & $48.8 \pm 7.6$ \\
\hline & Median & 54.0 & 53.5 & 49.9 & 50.2 \\
\hline
\end{tabular}

statistically significant differences within gender groups compared: ${ }^{*}-<0.05 ; * *-p<0.01$ $* * *-p<0.001$

$\% \mathrm{E}$ - percentage of energy

\section{DISCUSSION}

The increasing incidence of depressive disorders is an important social, economic and health issue, lowering the quality of life and worsening or limiting functioning of individuals.
Many studies have shown that social problems, external environment stressors, are more common in cities than rural areas. High population density urban areas are characterized by high rates of crime, mortality, social isolation, environmental pollution and noise $[9,10,19]$. Research shows that more than half of the world population today live in urban areas, and demographic forecasts predictits continuous growth to more than 6.3 billion by 2050 [19]. In Poland, $61 \%$ of people live in urbanized areas and the number is still increasing [2].

According to the available literature, depression can lead to negative life style behaviors (avoiding physical exercise), eating disorders including anorexia, bulimia, night eating syndrome [20]. Studies have shown that people with depression may display reduced, unchanged or increased appetite. It has been noted that the weight gain due to increased appetite and decreased physical activity occurs in approximately $40 \%$, whereas body weight reduction in $30 \%$ of patients with depression [20].

Other researchers emphasize that dietary habits of patients suffering from depression are frequently characterized by improper proportions in the intake of respective nutrients. The most common errors reported include eating foods with high glycemic index, incorrect diet composition in terms of fatty acids, macro- and micronutrients, abnormal relationship between $\mathrm{Na} / \mathrm{K}$ and too low fiber content $[20,21$, 22]. Our assessment of dietary intake of energy and essential components also revealed many irregularities.

In our study, women in both groups failed to meet dietary recommendations for the majority of nutrients, except for total protein and saturated fatty acids, which were consumed in excess. However, meansupply of most nutrients was higher in the food rations of rural women. In men, food rations of the urban population had higher nutrient content, although both groups were characterized by too low supply of fatty acids, both monounsaturated and polyunsaturated, and too high supply of saturated fatty acids.

Data obtained by other authors show that food rations consumed by people suffering from depression are often characterized by improper structure of fatty acids, with excessive share of SFA [21]. However, proper supply of fatty acids, especially the omega- 3 unsaturated fatty acids, EPA (eicosapentaenoic acid) and DHA (docosahexaenoic), may have a beneficial effect on the treatment of depressive patients. High consumption of fish as a source of unsaturated fatty acids has been found to correlate with the low percentage of patients with mental disorders in the population [20].

It has been shown that daily intake of omega- 3 fatty acids (1-2 g) is sufficient for healthy people, whereas their supply amounting up to $9.6 \mathrm{~g}$ appears safe and sufficient for patients with psychiatric disorders [22]. The antidepressant effect of EPA may be due to possible conversion to prostaglandins, leukotrienes and other compounds required for normal brain function. According to another theory, EPA and DHA influence signal transduction in brain cells through activation of PPAR receptors, inhibition of G-protein and of protein kinase $\mathrm{C}[20,21]$.

In the current study, the share of energy derived from the consumption of essential nutrients was found to deviate from the recommended standards in all the study groups. In a balanced food ration, protein-derived energy should not exceed $10-15 \%$, fat-derived energy should be lower than $30 \%$ and carbohydrate-derived energy should not be less 
than 55\% [18]. We demonstrated too high share of energy from fat (except for urban female residents), and very low from carbohydrates. Similar results were obtained by other authors, where the percentage of protein energy exceeded $17 \%$, fats-derived energy was over $30 \%$, and carbohydrate energy was lower than $50 \%$ [21]. Dietary habits of patients influenced their nutritional status.

In the present study, the nutritional status of patients was assessed by means of anthropometric measurements, body composition measurements and biochemical examinations. Body weight as a basic parameter for energy-protein nutritional status is one of the most important prognostic factors in the course of many diseases. Obesity is a known risk factor not only for depression, but also cardiovascular disease; it promotes development of carbohydrate and lipid disorders, and hypertension.

In our study, the average body mass index differed significantly only in women, and was statistically significantly lower for urban residents, with $50 \%$ of the subjects having normal BMI. In the remaining groups, the percentage of excessive body weight was found in $70 \%$ of female rural inhabitants and in over $80 \%$ in the male groups. Other authors have also demonstrated a significant percentage of depressive patients with BMI exceeding $25 \mathrm{~kg} / \mathrm{m}^{2}[5,23$, $24,25]$.

It has been shown that depressivesymptomsandBMIare inverselycorrelated with each other, thus indicating agreaterprotectiveshare of physiological, functional and psychological factors as compared to the amountof muscle massand increasedphysical activity. This wasreflected in Ahmadi's research, inwhich patientswith depression were characterized by an increased thickness of skinfolds and total body fat [23].

Waist circumferenceexceeding $80 \mathrm{cmin}$ womenand $94 \mathrm{~cm}$ in men (abdominalfat distribution) is an alarming trend observedin this study[15]. Waist circumferenceconfirming the accumulation of visceral fatin female patientswith depression $(>90 \mathrm{~cm})$ has also been reported by otherauthors $[5,25]$. In the present study, the averagefat content was lowerin female and male urban residents (30\% and $29 \%$, respectively) compared to female and male village inhabitants (37\% and 30\%, respectively), although statistically significant difference was found onlybetween women.

Other authors reported higherbody fat massinwomen(above $38 \%$ ) and lower in men (27\%) than those obtained in the present study $[24,25]$. The analysis of bodycomposition includedthe content ofvisceral adipose tissue andsubcutaneousadipose tissue. Althoughthere were nostatistically significant differencesbetween the comparedgroups, it was demonstratedthatVAT values in men and womenfrom the city were lowerthanin those from rural areas. The content ofSATwasat a similar levelin both gender groups.

Other authors reported lower average VAT $\left(94.6 \mathrm{~cm}^{2}\right)$ and a higher proportion of SAT $\left(389.5 \mathrm{~cm}^{2}\right)$ in comparison with those found in the present study [5]. In patients with depression, waist circumference was strongly correlated with both subcutaneous, $r=0.83$ and visceral adipose tissue, $r=0.77, p<0,001$ ) [5]. It has been demonstrated that visceral adipose tissue is metabolically active, associated with the secretion of pro-inflammatory factors (Interleukin 6, IL-6), tumor necrosis factor a (TNFa), adipocytokines, hemostasis and fibrinolysis markers (including plasminogen activator inhibitor-1, PAI-1) and growth factors (vascular endothelial growth factor, VEGF). The increase in intraabdominaladipose tissue in patients with depression may be associated with a greater risk for cardiovascular diseaseand diabetes $[5,15]$. Everson-Rose et al. showed that postmenopausal women, whose body fat exceeded $163 \mathrm{~cm}^{2}$ were 4 to 5 times more likely to have an adverselipidprofile (low HDL-cholesterol, hypertriglyceridemia, high ratio of LDL to HDL-cholesterol, hyperinsulinemia) [5]

In the current study, selected lipid parameters (total cholesterol, HDL-cholesterol, LDL-cholesterol) and blood glucose levels were consistent with the recommended standards in most male and female patients living in the city as opposed to the rural population. However, a considerable percentage of patients with abnormal lipid and carbohydrate parameters in all the study groups, together with persisting improper dietary habits, indicates a risk of metabolic diseases in the future.

\section{CONCLUSIONS}

The current study indicates abnormal eating habits of patients with depression, regardless of place of residence, coexisting with overweight and obesity. Food rations of patients were characterized by nutritional imbalance, especially by high supply of protein and saturated fatty acids. Residents of rural areas compared to urban residents had higher body weight, BMI, total body fat and a higher proportion of visceraladipose tissue. A larger percentage of rural than urban residents were characterized by disturbances in lipid and carbohydrate metabolism.

Improvement indietary habits, especially balanced intake of fatty acids, increased supply of mono- and polyunsaturated fatty acids and fiber, and reduced supply of protein products and saturated fatty acids can be beneficial for maintenance of good health. Proper health education, including the optimum selection of food products is necessary for patients with depression, especially from rural areas.

\section{REFERENCES}

1. Huot KL, Lutfiyya MN, Akers MF, Amaro ML, Swanoski MT, Schweiss SK. A population-based cross-sectional study of health service deficits among U.S. adults with depressive symptoms. BMC Health Serv Res. 2013; 13: 160 doi: 10.1186/1472-6963-13-160.

2. World Health Organization. Depression. http://www.who.int/mental health/management/depression/definition/en/idex1.html (access: 2012 October).

3. O'Connor EA, Whitlock EP, Gaynes B, Beil TL. Screening for depression in adults and older adults in primary care: an updated systematic review. Evidence Synthesis No.75. AHRQ Publication No. 1-05143-EF1. Rockville, Maryland Agency for Health Research and Quality, 2009.

4. Kim D. Blues from the Neighborhood? Neighborhood characteristics and depression. Epidemiol Rev. 2008; 30: 101-117.

5. Everson-Rose SA, Lewis TT, Karavolos K, Dugan SA, Wesley D, Powell LH. Depressive symptoms and increased visceral fat in middle-aged women. Psychosom Med. 2009; 71(4): 410-416.

6. Murabito JM, Massaro JM, Clifford B, Hoffmann U, Fox CS. Depressive symptoms are associated with visceral adiposity in a community-based sample of middle-aged women and men. Obesity. 2013;21(8): 1713-1719.

7. Vogelzangs N, Seldenrijk A, Beekman TF, van Hout HPJ, de Jonge P, Penninx B. Cardiovascular disease in persons with depressive and anxiety disorders. J Affect Disord. 2010; 125: 241-248.

8. Dziemidok P, Makara-Studzińska M, Jarosz MJ.Diabetes and depression: a combination of civilization and life-style diseases is more than simple problem adding-literature review. Ann Agric Environ Med. 2011; 18(2): 318-322. 
9. Heinz A, Deserno L, Reininghaus U. Urbanicity, social adversity and psychosis. World Psych. 2013; 12: 187-197.

10. Peen J, Schoevers RA, Beekman AT, Dekker J. The current status of urban-rural differences in psychiatric disorders. ActaPsychiatr Scan. 2010; 121: 84-93.

11. Ślusarz R, Borzyszkowska A, Szrajda J, Fidecki W, Haor B. Influence of selected socio-demographic factors on incidence of depressive disorders in women. Nursing Topics 2011; 19(1): 21-26.

12. World Health Organization. International statistical classification of diseases and health-related problems. 10th rev. Geneva, WHO, 1992.

13. Hamilton M. A rating scale for depression. J NeurolNeurosurg Psychiatry.1960; 23: 56-62.

14. Beck AT, Ward CH, Mendelson M, Mock J, Erbaugh J. An inventory for measuring depression. Arch Gen Psychiatry. 1961; 4(6): 561-571.

15. Alberti KG, Eckel RH, Grundy SM, Zimmet PZ, Cleeman JI, Donato KA, FruchartJCh, James WP, Loria CM, Smith SC. Harmonizing the metabolic syndrome: a joint interim statement of the international diabetes federation task force on epidemiology and prevention; National Heart, Lung, and Blood Institute; American Heart Association; World Heart Federation; International Atherosclerosis Society; and International Association for the study of Obesity. Circulation.2009; 120: $1640-1645$.

16. Perk J. European resolution on action to tackle cardiovascular diseases in clinical practice in 2012. Kardiol Pol. 2012; 70: S1-S100.

17. Szponar L, Wolnicka K, Rychlik E. A photograph album of products' various portion sizes.Institute of Food and Nutrition, Warsaw, 2000.
18. Jarosz M (ed.). Revised dietary norms for the Polish population, Institute of Food and Nutrition, Warsaw 2012.

19. McKenzie K, Murray A, Booth T. Do urban environments increase the risk of anxiety, depression and psychosis? An epidemiological study. J Affect Disord. 2013; 150: 1019-1024.

20. Hidaka BH. Depression as adisease of modernity: Explanations for increasing prevalence. J Affect Disord. 2012; 140: 205-214.

21. Appelhans BM, Whited MC, Schneider KL, Ma Y, Oleski JL, Merriam PA, Waring ME, Olendzki BC, Mann DM, Ockene IS.Depression severity, diet quality, and physical activity in women with obesity and depression. J AcadNutr Diet. 2012; 112: 693-698.

22. Sanhueza C, Ryan L, Foxcroft DR. Diet and the risk of unipolar depression in adults: systematic review of cohort studies. J Hum Nutr Diet. 2013; 26(1): 56-70.

23. Ahmadi SM, Mohammadi MR, Mostafavi SA, Keshavarzi S, Joulaei H, Sarikhani Y, Peimani P, Heydari ST, Lankarani KB.Dependence of the geriatric depression on nutritional status and anthropometric indices in elderly population.Iran J Psychiatry. 2013; 8(2): 92-96.

24. Valentine RJ, McAuley E, Vieira VJ, Baynard T, Hu L, Evans E, Woods JA. Sex differences in the relationship between obesity, C-reactive protein, physical activity, depression, sleep quality and fatigue in older adults. Brain, Behav Immun. 2009; 23(5): 643-648.

25. Williams LJ, Pasco JA, Henry MJ, Jacka FN, Dodd S, Nicholson GC, Kotowicz MA, BerkM. Lifetime psychiatric disorders and body composition: A population-based study. J Affect Disord. 2009; 118(1-3): 173-179. 